INRA Prod. Anim., 1999, 12 (4), 265-271
D. HERMIER ${ }^{1}$, M.R. SALICHON ${ }^{2}$,

G. GUY ${ }^{3}$, R. PERESSON ${ }^{2}$, J . MOUROT ${ }^{4}$, S. LAGARRIGUE ${ }^{5}$

${ }^{1}$ I NRA-Uni versi té Paris XI, Laboratoi re associ é de Physi ologi e de la Nutriti on, Bât 447, 15 rue Georges Clémenceau, 91405 Orsay Cedex

2 INRA Stati on de Recherches Avi coles, 37380 Nouzilly

${ }^{3}$ INRA Stati on Expérimentale des Palmi pèdes à Foi e gras, Arti guères, 40280 Benquet

${ }^{4}$ INRA Stati on de Recherches Porci nes, 35590 Lhermi tage

${ }^{5}$ INRA Laboratoi re de Généti que 65 rue de Sai nt-Bri euc, 35042 Rennes

email : domini quehermi er@i bai c.u-psud.fr

\section{La stéatose hépatique des palmipèdes gavés: bases métaboliques et sensibilité génétique}

Le gavage des palmipèdes pour la production de foie gras utilise leur capacité naturelle de synthèse et de stockage de lipides dans le foie. L'aptitude à produire du foie gras varie selon les races, en fonction du potentiel de synthèse hépatique et/ou des voies métaboliques empruntées par les lipides synthétisés: stockage dans le foie ou sécrétion vers les tissus périphériques.

L'état de stéatose hépatique traduit l'accumulation de lipides dans le foie. Chez les animaux sauvages, cette accumulation est une

\section{Résumé}

Chez les Palmipèdes, l'induction contrôlée d'une stéatose hépatique par gavage permet la production de foie gras. Or, la réponse métabolique au gavage est très variable et dépend, entre autres facteurs, de l'espèce ou de la race, ce qui laisse supposer un déterminisme génétique. Après une revue prenant en compte les éléments les plus récents disponibles sur la stéatose de gavage et son mécanisme, cet article décrit les études menées sur les oies Landaises (très sensibles à la stéatose hépatique) et sur les oies Polonaises (partiellement résistantes). Avant gavage, il existe très peu de différence dans le métabolisme lipidique entre les deux races. E $n$ revanche, durant un gavage à ingéré égal d'aliment, les oies Polonaises réagissent par une exportation plus efficace, sous forme de lipoprotéines, de leurs triglycérides hépatiques, mais aussi des phospholipides et des acides gras polyinsaturés qui leurs sont associés. Ainsi, comparées aux oies Landaises, elles montrent un engraissement périphérique plus important. Les oies Landaises associent une lipogenèse hépatique plus intense à une meilleure rétention des triglycérides et, probablement, de la phosphatidylcholine et des acides gras polyinsaturés, ce qui permet I'hypertrophie cellulaire par synthèse membranaire, et donc se traduit par une stéatose hépatique plus importante. adaptation physiologique aux besoins énergétiques dans des situations bien particulières : migration, hibernation. Chez les animaux domestiques, comme chez l'Homme, la stéatose hépatique est une situation indésirable voire pathologique. Seule exception : chez les palmipèdes, l'induction maîtrisée d'une stéatose hépatique est à l'origine de la production du foie gras.

En F rance, les contraintes économiques de cette production sont de trois ordres:

- le gavage doit être à la fois rapide et intense, afin d'obtenir le plus rapidement possible un foie gras de taille commercialisable (aspects quantitatifs) ;

- les modifications du foie doivent satisfaire aux critères technologiques et organoleptiques qui définissent un produit de qualité et qui conduisent, par exemple, à rejeter des foies gras amers ou trop fondants (aspects qualitatifs) ;

- la production du foie gras doit prendre en compte une législation européenne de plus en plus contraignante, qui vise à interdire le gavage, celui-ci étant jugé incompatible avec 
le bien-être des animaux (aspects éthiques).

La maîtrise de ces différents aspects repose sur une meilleure connaissance du métabolisme lipidique chez les oiseaux destinés au gavage. L'existence d'une sensibilité génétique à la stéatose hépatique a permis la mise en place de telles études chez l'oie. Les résultats de ces études sont replacés dans le contexte des données de la bibliographie.

\section{1 / La stéatose hépatique de gavage}

Selon les termes de la définition de Labie et Tournut cités par Salichon (1991), le foie gras des palmipèdes gavés est l'expression d'une stéatose hépatique d'origine nutritionnelle, hypertrophique et réversible. Cette stéatose est extrême puisque, chez l'oie, le poids du foie peut être multiplié par 10 en 15 jours, passant de $100 \mathrm{~g}$ à $1 \mathrm{~kg}$, et représenter jusqu'à $10 \%$ du poids corporel (Hermier et al 1994).

\section{1 / Composition du foie gras}

Le degré de stéatose est, lui aussi, exceptionnel puisque, dans un foie gras de bonne qualité, les lipides représentent plus de la moitié du poids, contre moins de $5 \%$ chez les oiseaux non gavés. En réponse au gavage, les lipides de toutes les classes s'accumulent dans le foie, mais les triglycérides prédominent ( $95 \%$ des lipides). Ces triglycérides sont riches en acides gras monoinsaturés, avec plus de $50 \%$ d'acide oléique, mais contiennent très peu d'acides gras polyinsaturés, qui appartiennent quasi exclusivement à la série n-6 (Gabarrou et al 1996, Fournier et al 1997). Enfin, il faut souligner la richesse du foie gras en cholestérol (parfois plus de $1 \mathrm{~g}$ pour $100 \mathrm{~g}$ ). L'accumulation des lipides se fait au détriment de l'eau, mais aussi des protéines, qui représentent moins de $10 \%$ du poids. Ainsi, trop riche en calories et en cholestérol, et pauvre en protéines et en acides gras essentiels, le foie gras est un aliment très déséquilibré sur le plan nutritionnel, mais dont la contribution à l'équilibre alimentaire reste anecdotique, du moins sur la plus grande partie du territoire français.

\section{2 / Aspects cellulaires}

Lors d'un gavage bien conduit, les lésions macroscopiques sont normalement inexistantes, et un foie de bonne qualité ne présente ni hémorragies, ni zones de nécrose, ni tâches verdâtres (cholestase). La trame conjonctive, réduite chez les oiseaux, est distendue à l'extrême, mais la capsule de Glisson qui entoure le foie reste intacte.

La surcharge lipidique touche les hépatocytes et ne semble pas concerner les autres types cellulaires (Labie et Tournut 1970). L'augmentation du rapport ARN/ADN traduit l'augmentation des synthèses protéiques à l'origine, d'une part, des enzymes de la lipogenèse et, d'autre part, des synthèses membranaires (Blum et Leclercq 1973). Les syn- thèses membranaires traduisent essentiellement une hypertrophie cellulaire, durant laquelle l'infiltration lipidique débute en zone périportale, pour gagner progressivement l'espace centrolobulaire (Labie et Tournut 1970, Bénard et Labie 1998). II ne semble pas y avoir d'hyperplasie cellulaire (Bénard et Labie 1998).

L'hypertrophie cellulaire, quoique énorme, reste réversible : à l'issue du gavage, des oies replacées dans leurs conditions initiales d'élevage peuvent jeûner spontanément pendant plusieurs jours. Au bout de quelques semaines, elles présentent une normalisation du poids et de la composition du foie, ainsi que des paramètres sanguins (Ivorec-Szylit et Szylit 1969, Babilé et al 1998). Des observations analogues ont été rapportées sur des canards soumis à trois cycles successifs de gavage et de repos (Bénard et al 1998).

Ainsi, la stéatose hépatique de gavage est très différente des autres stéatoses rencontrées chez les mammifères et même chez les oiseaux, telles que le syndrome "foie gras hémorragique" de la poule pondeuse, qui est une authentique pathologie (Hansen et Walzem 1993). En fait, dans le cas des palmipèdes d'élevage, le gavage exploite à son maximum les capacités de stockage hépatique des lipides rencontrées chez les oiseaux migrateurs. Dans ces espèces, une stéatose hépatique physiologique, quoique modérée, permet la fourniture d'énergie pendant les jours de migration (Bénard et Labie 1998). Cette capacité d'adaptation naturelle du métabolisme lipidique permet d'expliquer à la fois l'intensité de la stéatose hépatique de gavage et sa réversibilité, dans des conditions certes extraphysiologiques, mais non pathologiques.

\section{3 / Facteurs de variation}

\section{a / Quantitatifs}

Pour les raisons évoquées ci-dessus, les palmipèdes, c'est-à-dire les différentes espèces d'oies et de canards, sont actuellement les seuls oiseaux domestiques gavés pour la production de foie gras. Cependant, même au sein des palmipèdes, l'intensité de la stéatose hépatique varie en fonction des espèces (tableau 1). En France, la production commerciale du foie gras repose sur l'oie (Anser anser) de race Landaise, le canard de Barbarie (Cairina moschata) et, surtout, sur le canard Mulard, hybride intergénérique de la cane commune de type Pékin (Anas platyrinchos) et du canard de B arbarie. Les autres types d'oies ou de canards, destinés à la production de viande, développent des stéatoses hépatiques réduites, et ce en dépit de poids corporels ou d'ingérés en gavage comparables (Hermier et al 1991). Même chez les races sensibles à la stéatose hépatique, la variabilité de la réponse au gavage est souvent considérable. Ainsi, lors d'une expérience conduite chez l'oie Landaise, le poids moyen des foies gras était d'environ $1000 \mathrm{~g}$, avec des valeurs extrêmes de $300 \mathrm{~g}$ et de $1500 \mathrm{~g}$ (Hermier et al 1994). 
Ainsi, au-delà des capacités naturelles de stockage hépatique des lipides, il existe une sensibilité génétique à la stéatose hépatique de gavage, accentuée par des générations de sélection.

\section{b / Qualitatifs}

La fonte lipidique est le principal critère de qualité technologique du foie gras. Le terme traduit l'exsudation extrahépatique des lipides sous l'effet de la chaleur, qui survient lors des traitements de conservation thermique (pasteurisation et stérilisation). Ce phénomene résulte de la fragilisation des membranes plasmiques, distendues sous l'effet de I'hypertrophie cellulaire. Ces membranes se rompent et libèrent une partie des triglycérides cytoplasmiques, qui vont se solidifier autour du foie gras. Pour des raisons esthétiques et organoleptiques, cette fonte lipidique est très préjudiciable à la qualité du foie gras. De surcroît, les foies gras les plus gros, donc les plus stéatosés, seront très pénalisés.

Là encore, il existe une forte composante génétique, qui se traduit par une fonte lipidique énorme chez le canard de Barbarie, alors que son foie gras est le plus léger (tableau 1). Une étude récente a montré que, chez l'oie, le gavage entraînait un appauvrissement des membranes plasmiques hépatiques en cholestérol et en phospholipides (Salichon et al 1994). Cependant, rien ne permet actuellement de faire le lien entre les caractéristiques membranaires et la variabilité génétique de la fonte lipidique.

\section{2 / Mécanisme de la stéatose hépatique de gavage}

\section{1 / Particularités du métabolisme lipidique des oiseaux}

Chez les oiseaux, les lipides alimentaires, après leur absorption dans l'intestin grêle, sont assemblés dans les entérocytes sous forme de portomicrons et libérés dans la circulation porte (Bensadoun et Rothfield 1972). Les portomicrons sont des lipoprotéines très proches des chylomicrons des mammifères quant à leur composition, mais leur parcours métabolique est différent, puisque les portomicrons vont traverser le foie avant de rejoindre la circulation générale. Ainsi, les lipides qu'ils transportent vont pouvoir être captés et utilisés par le foie dès ce premier passage. Ceci est particulièrement important pour les acides gras polyinsaturés essentiels (dans l'aliment de gavage, composé essentiellement de maïs, l'acide linoléique, C 18:2 $n-6$, représente plus de la moitié des acides gras).

Par ailleurs, chez les oiseaux, le foie est le site principal de la lipogenèse de novo (Léveille et al 1975). Comme chez les mammifères, la synthèse des acides gras dépend de la disponibilité de précurseurs glucidiques alimentaires. Elle est donc fortement stimulée en période postprandiale, qui correspond en même temps à une intense stimulation par l'insuline des principales enzymes de la lipogenèse chez les oiseaux, à savoir l'enzyme

Tableau 1. Production du foie gras chez les palmipèdes (Salichon et al 1994).

\begin{tabular}{|l|c|c|c|}
\hline & Oie Landaise & Canard Mulard & Canard de Barbarie \\
\hline Poids corporel $(\mathrm{kg})$ & 7,4 & 6,5 & 6,5 \\
Poids de foie gras $(\mathrm{g})$ & 770 & 680 & 550 \\
Fonte lipidique (\%) & 21,2 & 43,9 & 55,6 \\
\hline
\end{tabular}

\section{4 / Bilan}

La réponse du foie au gavage, qu'elle soit quantitative ou qualitative, est sous le contrôle, entre autres paramètres, de facteurs génétiques dont la nature reste à déterminer. L'existence, à I'INRA (Station Expérimentale des Palmipèdes à Foie gras, Artiguères), de types d'oies présentant des aptitudes de production différentes (foie gras ou viande) nous a fourni une base originale pour comparer la réponse au gavage de types génétiques différents dans des conditions expérimentales contrôlées : élevage et gavage en parallèle, aux mêmes âges, dans les mêmes conditions d'ambiance et d'alimentation.

Cette étude s'est appuyée sur les connaissances générales du métabolisme lipidique des oiseaux ainsi que sur les données acquises précédemment sur le métabolisme lipidique spécifique de l'oie (voir en particulier Hermier et al 1988, 1991 et 1994). malique et la synthétase des acides gras (Goodrige 1987). Le foie des oiseaux est le site principal de la synthèse des triglycérides, mais aussi, comme chez les mammiferes, des phospholipides et du cholestérol.

Les lipides hépatiques sont assemblés par le foie et sécrétés sous forme de lipoprotéines, la fraction protéique des lipoprotéines étant elle aussi synthétisée par le foie. Chez les oiseaux immatures, y compris chez l'oie, il s'agit essentiellement de l'apoB et de I'apoA-I (Hermier et al 1988). Les triglycérides s'associent préférentiellement à l'apoB pour former des VLDL (very low density lipoproteins), tandis que la majorité du cholestérol et des phospholipides s'associent à l'apoA-I pour former des HDL (high density lipoproteins). L'assemblage des lipoprotéines débute dans le réticulum endoplasmique et se poursuit dans l'appareil de Golgi, puis les particules naissantes sont incluses dans des vésicules de sécrétion et dirigées vers la membrane plasmique. 
Comme nous le verrons dans le cas du gavage, les processus d'assemblage et de sécrétion des lipoprotéines peuvent se révéler insuffisamment rapides pour traiter tous les triglycérides synthétisés en période postprandiale. Ces triglycérides excédentaires seront stockés dans des vésicules cytoplasmiques temporaires. Ils ne pourront être rediriges vers le circuit de sécrétion qu'après hydrolyse partielle suivie de réestérification (Mooney et Lane 1981). Enfin, l'assemblage et la sécrétion d'une particule de lipoprotéines imposent que ses différents constituants lipidiques et protéiques soient disponibles simultanément. Ainsi, la sécrétion des triglycérides sous forme de VLDL dépend de la présence en quantité suffisante de cholestérol, de phospholipides, et d'apoB. Une fois libérés dans la circulation, les triglycérides des VLDL (ainsi que ceux des portomicrons) sont hydrolysés par la lipoprotéine lipase, tout particulièrement du tissu adipeux, la plus active en période postprandiale. Comme la lipogenèse est très limitée dans le tissu adipeux des oiseaux (Léveille et al 1975), la croissance de ce tissu dépend essentiellement du captage et de la réestérification des acides gras apportés par les VLDL.

\section{2 / E ffet du gavage}

L'aliment de gavage consiste essentiellement en une pâtée de maîs et d'eau. C'est donc un aliment très énergétique ( 3250 $\mathrm{kcal} / \mathrm{kg})$, riche en glucides facilement assimilables (amidon), mais pauvre en lipides (environ $4 \%$ ). Comme nous l'avons vu précédemment, ce type d'aliment stimule fortement la lipogenèse endogène et la production des lipoprotéines hépatiques, dont les concentrations plasmatiques s'élèvent considérablement sous l'effet du gavage (tableau 2). Curieusement, et bien que cette observation ne soit pas systématique, ces lipoprotéines contiennent anormalement peu de triglycérides. Un défaut d'assemblage des triglycérides aux lipoprotéines serait alors un facteur favorisant la stéatose hépatique. Quoiqu'il en soit, et malgré l'augmentation de la concentration des lipides sanguins, les lipides de

Tableau 2. Modifications lipidiques en réponse au gavage chez l'oie Landaise (Hermier et al 1991 et 1994).

\begin{tabular}{|l|c|c|c|}
\hline & Témoins & & Gavées \\
\hline Plasma & & & \\
VLDL (g/l) & 0,76 & $* * *$ & 2,19 \\
Triglycérides des VLDL (\%) & 42,7 & $* * *$ & 29,1 \\
HDL (g/l) & 4,89 & $* * *$ & 11,31 \\
Triglycérides des HDL (\%) & 6,9 & $*$ & 4,4 \\
\hline Foie & & & \\
Poids (g) & 97 & $* * *$ & 819 \\
Eau (\%) & 68,5 & $* * *$ & 34,6 \\
Lipides (\%) & 4,2 & $* * *$ & 54,4 \\
Triglycérides (g/foie) & 1,7 & $* * *$ & 418,0 \\
Phospholipides (g/foie) & 1,96 & $* * *$ & 19,30 \\
Cholestérol libre (g/foie) & 0,35 & $* * *$ & 4,02 \\
Esters de cholestérol (g/foie) & 0,21 & $* * *$ & 6,06 \\
\hline
\end{tabular}

$*: \mathrm{P}<0,05 ; * * *: \mathrm{P}<0,001$ toutes les classes s'accumulent dans le foie des oiseaux gavés, avec une prédominance des triglycérides, mais aussi des esters de cholestérol, qui sont l'autre forme de stockage (tableau 2). Les quantités de phospholipides et de cholestérol libre, qui sont avant tout des lipides membranaires de structure, sont elles aussi très augmentées, mais dans des proportions moindres. Les raisons pour lesquelles les triglycérides néosynthétisés sont dirigés vers le stockage cytoplasmique plutôt que vers la sécrétion restent hypothétiques. D'une part, les capacités de synthèse de l'apoB, protéine majeure des VLDL, ou de la MTP (microsomal triglyceride transfer protein), qui permet l'assemblage des VLDL, peuvent devenir limitantes. D'autre part, les triglycérides stockés dans les vésicules cytoplasmiques ont tendance à y rester : en effet, lors du gavage, les animaux sont en permanence sous l'effet d'une stimulation insulinique postprandiale, ce qui empêcherait les processus d'hydrolyse et de réestérification nécessaires à leur mobilisation. Les oiseaux n'étant jamais à jeun, le foie ne peut sécréter cet excès de triglycérides, qui continuent de s'accumuler. comme nous l'avons vu précédemment, le phénomène est réversible puisque l'arrêt du gavage permet la mobilisation des lipides hépatiques et le retour à la normale.

II n'en reste pas moins que les oies répondent au gavage par un effort considérable de sécrétion des triglycérides hépatiques (tableau 2), ce qui provoque un engraissement périphérique considérable, qui contribue à la valeur commerciale et aux qualités organoleptiques des animaux gavés (magret, confit).

\subsection{Influence de l'origine génétique}

Des oies Landaises, dont l'aptitude à la production de foie gras n'est plus à démontrer, ont été comparées à des oies Polonaises, normalement élevées pour la production de viande. Les essais successifs ont été réalisés avec des oies des deux races, mâles, nées le même jour, élevées et gavées dans les mêmes conditions à la Station Expérimentale des Palmipèdes à Foie gras (INRA, Artiguères). L'objectif était d'évaluer la répartition des lipides d'origine hépatique dans les différents compartiments corporels, chez des animaux témoins ou gavés.

\section{a / Composition corporelle}

En réponse au gavage, l'oie Landaise développe un foie gras deux fois plus lourd que I'oie Polonaise, et ce malgré des quantités similaires d'aliment ingéré en gavage (tableau 3). A l'inverse, la stéatose périphérique est plus prononcée chez l'oie Polonaise, que ce soit pour les tissus adipeux viscéraux (gras abdominal) ou sous-cutanés (ensemble peau + gras associé au magret). Enfin, l'oie Polonaise exprime son aptitude à la production de viande par un plus grand développement du muscle du magret (Pectoralis major). Cette analyse globale de la composi- 
tion corporelle indique déjà que les lipides synthétisés par le foie en réponse au gavage sont stockés préférentiellement in situ chez I'oie Landaise, et exportés vers les tissus adipeux chez l'oie Polonaise.

\section{b / Composition du foie}

Chez les oies non gavées, le poids du foie et sa composition sont identiques dans les deux races, et similaires aux valeurs figurant au tableau 2. Les phospholipides représentent plus de $80 \%$ des lipides, et sont constitués majoritairement de phosphatidylcholine $(70 \%)$ et de phosphatidyléthanolamine (25\%). Sous l'effet du gavage, le poids plus élevé du foie chez l'oie Landaise s'explique par une accumulation plus importante de lipides. Par conséquent, les proportions des autre constituants hépatiques sont plus faibles chez les oies Landaises, alors que les quantités (exprimées en g/foie) sont globalement identiques dans les deux races (tableau 4). Tous les lipides s'accumulent dans le foie des animaux gavés, et ce en quantités plus importantes chez les oies Landaises, à l'exception des phospholipides; mais ce sont surtout les triglycérides qui marquent la différence entre les deux races. Quant aux phospholipides, non seulement leur accumulation est identique dans les deux races (environ $3 \mathrm{~g} /$ foie avant gavage et $12 \mathrm{~g} /$ foie après gavage), mais leur composition reste identique à celle des animaux témoins.

Enfin, le profil des acides gras du foie gras est très voisin dans les deux races : les lipides neutres (essentiellement des triglycérides) contiennent surtout de l'acide oléique (18:1 $\mathrm{n}-9,52 \%)$ et de l'acide palmitique (16:0, 27$33 \%)$ tandis que les phospholipides contiennent principalement de l'acide oléique (30\%), de l'acide palmitique (21\%), de l'acide stéarique (18:0, $21 \%$ ) et de l'acide arachidonique $(20: 4 n-6,13 \%)$

\section{c / Lipides plasmatiques}

Chez les animaux non gavés, il existe très peu de différence dans le profil des lipoprotéines plasmatiques entre les deux races; cependant, on note chez l'oie Polonaise des concentrations plus élevées de HDL, principal transporteur des phospholipides (Fournier et al 1997).

Ainsi que cela a été mentionné précédemment (tableau 2), I'oie répond au gavage par une élévation des VLDL et des HDL. Cette élévation est plus marquée chez l'oie Polonaise, dont les lipides sont, de surcroît, plus riches en phospholipides (tableau 5). De plus, chez l'oie Polonaise, ces phospholipides de HDL sont plus riches en phosphatidylcholine (au détriment de la phosphatiyléthanolamine) et en acides gras polyinsaturés (au détriment des monoinsaturés) (tableau 6).

\section{Conclusion}

Les études réalisées sur les oies Landaises et Polonaises ont montré que la sensibilité à la stéatose hépatique s'exprimait, non pas par
Tableau 3. Composition corporelle des oies Landaises et Polonaises gavées (Fournier 1994).

\begin{tabular}{|l|c|c|c|}
\hline & Landaises & & Polonaises \\
\hline Poids à l'abattage $(\mathrm{kg})$ & 8,78 & $*$ & 8,28 \\
Foie $(\mathrm{g})$ & 1005 & $* * *$ & 485 \\
Tissu adipeux abdominal $(\mathrm{g})$ & 411 & $* *$ & 523 \\
Tissu adipeux sous-cutané $(\mathrm{g})$ & 142 & $*$ & 161 \\
Muscle Pectoralis major $(\mathrm{g})$ & 239 & $* *$ & 275 \\
\hline
\end{tabular}

$*: \mathrm{P}<0,05 ; * *: \mathrm{P}<0,01 ; * * *: \mathrm{P}<0,001$

Tableau 4. Composition (moyenne \pm écart type) du foie des oies Landaises et Polonaises gavées.

\begin{tabular}{|l|c|c|c|}
\hline & $\begin{array}{c}\text { Landaises } \\
\mathrm{n}=16\end{array}$ & & $\begin{array}{c}\text { Polonaises } \\
\mathrm{n}=13\end{array}$ \\
\hline Poids du foie (g) & $683 \pm 241$ & $* * *$ & $420 \pm 90$ \\
Eau (\%) & $38,7 \pm 6,0$ & $* * *$ & $52,4 \pm 9,6$ \\
Protéines (\%) & $8,7 \pm 1,7$ & $* * *$ & $13,2 \pm 0,9$ \\
Minéraux (\%) & $0,56 \pm 0,17$ & $* * *$ & $0,93 \pm 0,26$ \\
Lipides (\%) & $49,1 \pm 9,3$ & $* * *$ & $30,1 \pm 13,1$ \\
Triglycérides (g/foie) & $333 \pm 17$ & $* * *$ & $127 \pm 88$ \\
Phospholipides (g/foie) & $12,4 \pm 4,1$ & & $11,6 \pm 3,4$ \\
Cholestérol libre (g/foie) & $0,81 \pm 0,48$ & $*$ & $0,45 \pm 0,25$ \\
Esters de cholestérol (g/foie) & $3,30 \pm 1,99$ & $* *$ & $0,93 \pm 0,74$ \\
\hline
\end{tabular}

Tableau 5. Lipoprotéines plasmatiques des oies Landaises et Polonaises gavées (Fournier et al1997).

\begin{tabular}{|l|c|c|c|}
\hline & Landaises & & Polonaises \\
\hline VLDL (g/l) & 3,31 & $* *$ & 4,89 \\
Triglycérides des VLDL (\%) & 51,2 & & 48,5 \\
Phospholipides des VLDL (\%) & 16,0 & $* * *$ & 18,9 \\
HDL (g/l) & 9,47 & $* * *$ & 14,64 \\
Triglycérides des HDL (\%) & 6,96 & & 6,13 \\
Phospholipides des HDL (\%) & 22,5 & $*$ & 25,8 \\
\hline
\end{tabular}

$*: \mathrm{P}<0,05 ; * *: \mathrm{P}<0,01 ; * * *: \mathrm{P}<0,001$

Tableau 6. Composition des HDL (en \%, moyenne \pm écart type) chez les oies Landaises et Polonaises gavées.

\begin{tabular}{|c|c|c|c|}
\hline & $\begin{array}{c}\text { Landaises } \\
\mathrm{n}=16\end{array}$ & & $\begin{array}{c}\text { Polonaises } \\
n=13\end{array}$ \\
\hline $\begin{array}{l}\text { Phospholipides } \\
\text { Phosphatidyléthanolamine (PE) } \\
\text { Phosphatidylsérine + Phosphatidylinositol } \\
\text { Phosphatidylcholine (PC) } \\
\text { Sphingomyéline } \\
\text { PC /PE }\end{array}$ & $\begin{array}{l}7,09 \pm 2,11 \\
2,48 \pm 1,77 \\
87,4 \pm 3,5 \\
1,13 \pm 1,65 \\
13,6 \pm 4,7\end{array}$ & $\begin{array}{l}* * \\
* \\
* * *\end{array}$ & $\begin{array}{l}4,69 \pm 1,66 \\
2,14 \pm 0,95 \\
90,1 \pm 2,4 \\
1,30 \pm 0,82 \\
21,2 \pm 6,5\end{array}$ \\
\hline $\begin{array}{l}\text { Acides gras des phospholipides } \\
\text { Saturés } \\
\text { Monoinsaturés } \\
\text { Polyinsaturés }\end{array}$ & $\begin{array}{l}42,3 \pm 6,1 \\
30,4 \pm 7,6 \\
25,3 \pm 4,8\end{array}$ & $*$ & $\begin{array}{l}46,1 \pm 7,2 \\
23,1 \pm 3,4 \\
30,3 \pm 5,0\end{array}$ \\
\hline
\end{tabular}

$*: \mathrm{P}<0,05 ; * *: \mathrm{P}<0,01 ; * * *: \mathrm{P}<0,001$

des caractéristiques intrinsèques des paramètres lipidiques de base, puisqu'il n'y a que très peu de différences entre races chez les animaux témoins, mais à travers la capacité de réponse au gavage (figure 1 ). En particulier, l'oie Polonaise réagit par une mise en circulation plus efficace des triglycérides, mais aussi des phospholipides et des acides gras polyinsaturés. Ces composants seraient alors 
moins disponibles pour les synthèses membranaires nécessaires à l'hypertrophie cellulaire qui accompagne la stéatose hépatique. La sensibilité à la stéatose hépatique est ainsi régie par l'équilibre qui s'établit dans les hépatocytes entre la sécrétion et la rétention in si tu des lipides néosynthétisés. Par ailleurs, l'exportation plus efficace des triglycérides explique l'engraissement périphérique plus important de l'oie Polonaise.

Cependant, les études les plus récentes montrent que ce schéma n'est peut-être pas si simple. $\mathrm{E}$ effet, à ingéré égal, la lipogenèse hépatique est plus élevée chez l'oie Landaise, à en juger par une activité plus intense de l'enzyme malique et par une plus grande abondance de son ARNm (tableau 7). Or, l'enzyme malique est considérée comme une des enzymes-clés de la lipogenèse chez les oiseaux (Hermier 1997).

Quoiqu'il en soit, si la stéatose hépatique est avant tout le reflet du métabolisme des triglycérides, le degré de cette stéatose implique sans doute le métabolisme des phospholipides, et c'est dans ce domaine que les recherches doivent être poursuivies. Elles permettraient peut-être aussi d'expliquer l'altération de la qualité entraînée par la fonte lipidique, particulièrement pénalisante dans certains types génétiques (cf. tableau 1) Par ailleurs, I'hypothèse posée ci-dessus, à savoir que le niveau de production de foie gras

Tableau 7. Activité de l'enzyme malique chez les oies gavées.

\begin{tabular}{|l|c|c|c|}
\hline & Landaises & & Polonaises \\
\hline $\begin{array}{l}\text { Activité spécifique } \\
\text { ( } \mu \text { M de NADPH formé/min/mg protéines) }\end{array}$ & $8,60 \pm 1,72$ & $* * *$ & $5,53 \pm 1,68$ \\
Quantité d'ARNm (unités arbitaires) & $34,0 \pm 12,4$ & $* *$ & $21,9 \pm 10,1$ \\
\hline
\end{tabular}

dépend de l'équilibre entre stockage et sécrétion des lipides hépatiques, devra être rapidement testée chez le canard. En effet, la production du foie gras en France repose essentiellement sur le canard Mulard, dont le métabolisme lipidique est à peu près inconnu. Or, la mise en évidence de paramètres métaboliques indicateurs d'une plus ou moins grande sensibilité à la stéatose hépatique serait une aide précieuse à la sélection et/ou au tri précoce des animaux les plus aptes à la production de foie gras.

Figure 1. Métabolisme lipidique chez l'oie gavée.

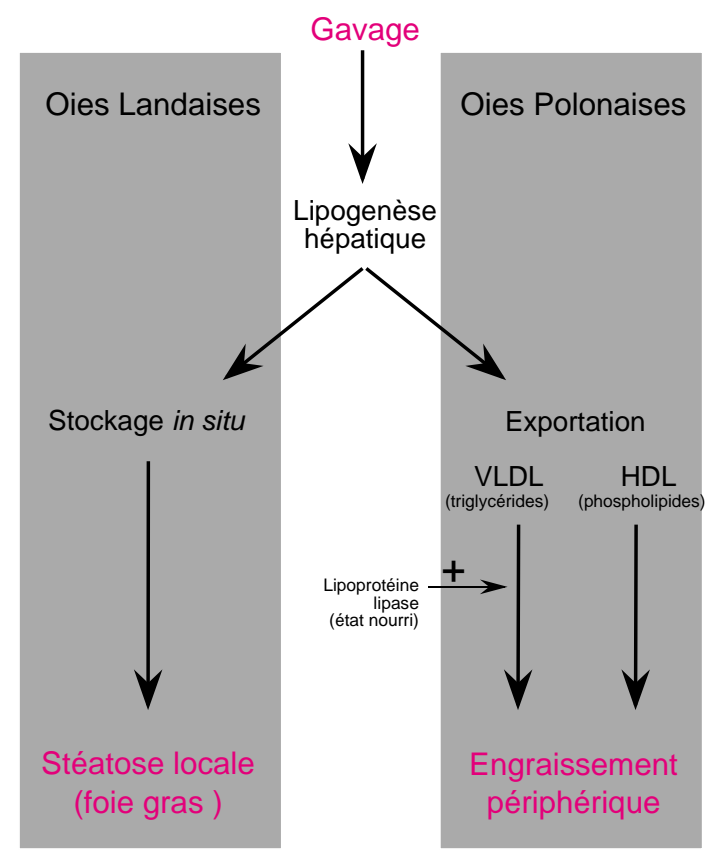

\section{Références}

Babilé R., Auvergne A., Dubois J .P., Bénard G., Manse H., 1998. Réversibilité de la stéatose hépatique chez l'oie. 3ème J ournées de la Recherche sur les Palmipèdes à $F$ oie gras, Bordeaux, 27 au 27 octobre, 45-48.

Bénard G., Labie Ch., 1998. Evolution histologique du foie des palmipèdes au cours du gavage. 3èmes J ournées de la Recherche sur les Palmipèdes à Foie gras, Bordeaux, 27 au 27 octobre, 31-36.

Bénard G., Bénard P., Prehn D., Bengone T., J ouglar J.Y., Durand S., 1998. Démonstration de la réversibilité de la stéatose hépatique obtenue par gavage de canards mulards. Etude réalisée sur 3 cycles de gavage-dégavage. 3èmes journées de la Recherche sur les Palmipèdes à Foie gras, Bordeaux, 27 au 27 octobre, 49-52.

Bensadoun A., Rothfield A., 1972. The forms of absorption of lipids in the chicken, Gallus domesticus. Proc. Soc. Exp. Biol. Med., 148, 347-350.

Blum J.C., Leclercq B., 1973. Nouvelles précisions sur les modifications biochimiques et histologiques du foie provoquées par le gavage. Acti delle Giornate Avicole, Varese. II, 31, 193-207.
Fournier E., 1994. Comparaison du métabolisme lipidique chez deux races d'oies différant par leur aptitude à la stéatose hépatique. Mémoire de DEA de Sciences des Aliments, Université Bordeaux I.

Fournier E., Peresson R., Guy G., Hermier D., 1997. Relationships between storage and secretion of hepatic lipids in two breeds of geese with different susceptibility to liver steatosis. Poultry Sci., 76, 599-607.

Gabarrou J.F., Salichon M.R., Guy G., Blum J.C., 1996. Hybrid ducks overfed with boiled corn develop an acute hepatic steatosis with decreased choline and polyunsatured fatty acid level in phospholipids. Reprod. Nutr. Develop., 36, 473-484.

Goodridge A.G., 1987. Dietary regulation of gene expression : enzymes involved in carbohydrates and lipid metabolism. Annu. Rev. Nutr., 7, 157-185.

Hansen R.J ., Walzem R.L., 1993. Avian fatty liver hemorrhagic syndrome : a comparative review. Adv. Vet. Sci. Comp. Med., 37, 451-468.

Hermier D., 1997. Lipoprotein metabolism and fattening in poultry. J. Nutr., 127, 805S-808S 
Hermier D., Forgez P., Laplaud P.M., Chapman M.J ., 1988. Density distribution and physicochemical properties of plasma lipoproteins in the goose, Anser anser, a potential of liver steatosis. J. Lipid Res., 29, 893-907.

Hermier D., Saadoun A., Salichon M.R., Sellier N., Rousselot-Pailley D., Chapman M.J., 1991. Plasma lipoproteins and liver lipids in two breeds of geese with dif ferent susceptibility to hepatic steatosis : changes induced by development and force-feeding. Biochim. Biophys. Acta, 26, 331-339.

Hermier D., Rousselot-Pailley D., Peresson R., Sellier N 1994. Influence of orotic acid and estrogen on hepatic lipid storage and secretion in the goose susceptible to liver steatosis. Biochim. Biophys. Acta, 1211, 97-106.

Ivorec-Szylit O., Szylit M., 1969. Etude des lactidodéshydrogénases plasmatique et hépatique chez l'oie au cours d'une stéatose provoquée par gavage. Ann. Biol. Anim. Biochim., 9, 205-218.
Labie C., Tournut B., 1970. Recherches sur les modifications histologiques et biochimiques chez les oies soumises au gavage. Cah. Med. Vet., 39, 247-261.

Léveille G.A., Romsos D.R., Yeh Y.Y., O'Hea E.K., 1975. Lipid biosynthesis in the chick. A consideration of the site of synthesis, influence of diet and possible regulatory mechanisms. Poultry Sci., 54, 1075-7093.

Mooney R.A., Lane M.D., 1981. Formation and turn-over of triglyceride-rich vesicles in chick liver. J . Biol. Chem., 256, 11724-11733.

Salichon Y., 1991. Elevage et gavage des palmipèdes pour la production du foie gras. Armand Colin Editeur, Paris.

Salichon M.R., Guy G., Rousselot-Pailley D., Blum J.C., 1994. Composition des 3 types de foie gras : oie, canard mulard et canard de Barbarie. Ann. Zootech., 43, 213-220.

\section{Abstract}

Hepatic steatosis in waterfowl: metabolic basis and genetic susceptibility.

In waterfowl, the controlled induction of hepatic steatosis by overfeeding allows the production of "foie gras". However, metabolic response to overfeeding varies considerably and depends, among other factors on species or breed, which may be indicative of a genetic determinism. After a review that takes into account the most recent data available on overfeeding steatosis and its mechanism, the present article describes the studies conducted on the Landes geese (highly susceptible to hepatic steatosis) and the Poland geese (partly resistant). B efore overfeeding, there are very few differences in lipid metabolism of the two breeds of geese. By contrast, and despite the same food intake during overfeeding, the Poland geese respond by a more effec- tive exportation of hepatic triglycerides in lipoproteins, as well as phospholipids and associated polyunsaturated fatty acids (PUFAS). Thus, when compared to the Landes geese, they exhibit a greater extrahepatic fattening. The Landes geese exhibit a higher hepatic lipogenesis together with a better retention of triglycerides and, most probably, phosphatidylcholine and PUFAS, which allow membrane synthesis and cell hypertrophy, and result in a greater hepatic steatosis.

HERMIER D., SALICHON M.R., GUY G., PERESSON R., MOUROT J., LAGARRIGUE S., 1999. La stéatose hépatique des palmipèdes gavés : bases métaboliques et sensibilité génétique. INRA Prod. Anim., 12, 265-271. 